\title{
Ueber einen neuen Beleuchtungsapparat am Mikroskop.
}

\author{
Von \\ Dr. W. Wbe, \\ ao. Professor in Jena. \\ Hierzu ein Holzschnitt.
}

Die in der vorigen Abhandlung unter (11) aufgestellten theoretischen Sätze uber die Beleuchtung milkroskopischer Objecte haben mich schon vor mehreren Jahren auf die Construction eines Illuminators geführt, der - in allen Theilen rein nach theoretischen Erwägungen combinirt - durch seine den Consequenzen der Theorie durchaus entsprechende Leistung diese zugleich auf die einfachste Art praktisch bewährt. Ich habe ihn ursprünglich nur für meinen eigenen Gebrauch ausführen lassen und habe denselben vielfach zu den im vorigen Aufsatze beschriebenen Experimenten, namentlich aber zum Studium der Abbildungsfehler der Objective wach der in (10) erwähnten Methode benutzt. Da indess der A pparat durch Solche, die ihn bei mir gesehen, schon eine ziemliche Verbreitung unter praktischen Mikroskopikern gefunden hat ${ }^{1}$ ) und ich sonach annehmen darf, dass er auch für die gewöhnlichen Bedürfnisse der mikroskopischen Beobachtung unter Umständen von Nutzen sein kann, so erlaube ich mir, den Lesern dieses Archivs im Folgenden eine kurze Beschreibung desselben vorzulegen.

Das Prinzip, auf welches der Apparat gegründet ist, kann am

1) Von Herrn C. Zeiss in Jena ist die Vorrichtung unter die stän digen, Artikel seiner Werkstatt aufgenommen; Nr. 24 des Katalogs von 1872. 
einfachsten bezeichnet werden, wenn man die Wirkungsweise des gewöhnlichen Beleuchtungsspiegels zum Vergleich in Betracht zieht. Der bewegliche Spiegel am Mikroskop bezweckt, mit Hilfe einer Lichtquelle von gegebener Lage - z. B. einer hellen Wolke, einer weissen Wand oder dergl. - eine begrenzte leuchtende Fläche in beliebiger Lage unterhnlb des mikroskopischen Präparats herzustellen, welche, abgesehen von den Lichtverlusten durch die Reflexion, mit der Leuchtkraft der primären Lichtquelle das Präparat bestrahlt. Von welchen Bedingungen diese Uebertragung der Leuchtkraft auf die ganze Fläche des Spiegels abhängt, unter welchen Unständen und in wie weit dabei die Form der Spiegelfläche - ob concav oder plan - Einfluss gewinnt a. A. m., findet man bei Nägeli und Schwendener (Das Mikroskop, S. 85 u. f.) so vollständig und einleuchtend auseinandergesetzt, dass jede weitere Erklärung dartuber überflüssig ist. Hier sei nur zweierlei hervorgehoben:

Erstens, was den Vorzug dieser Beleuchtungsvorrichtung ausmacht - dass dieselbe, wie sie die einfrchste ist der Construction nach, auch unbedingt die wirksamste bleibt in Hinsicht auf die specifische Intensität der zu erzielenden Beleuchtung. Alle complicirteren Apparate, wie z. B. die - Iucus a non lucendo - "Condensor" benannten Linsencombinationen, führen unvermeidlicher Weise eine grössere Verminderung der in der Lichtquelle disponibeln Leuchtkraft herbei als der einfache Beleuchtungsspiegel, weil zu den Lichtverlusten durch die auf alle Fälle doch unentbehrliche Spiegelung noch diejenigen durch mehrfache Brechungen hinzutreten. Soll daher mit einem solchen zusammengesetzten Illuminator eine bestimmte Helligkeit des Bildes erzielt werden, so bedarf es stets der Anwendung eines Strahlenkegels von grösserem Divergenzwinkel d. h. der Herstellung einer lichtgebenden Fläche unter dem Object von grösserem angularen Durchmesser, als beim Gebrauch des einfachen Spiegels nöthig wäre; der Effect solcher Apparate ist also das directe Gegentheil von Demjenigen, was ihr Name besagen will, nicht eine Condensation, sonderu eine Verdu un nung des Lichts.

Zweitens, was den Nachtheil des gewöhnlichen Beleuchtungsspiegels ansmacht - dass die Bedingungen für die wïnschenswerthen Modificationen und für die sichere Regulirung der Beleuchtung beim Gebrauch desselben keineswegs besonders günstig stehen. Die Abstufungen in der Einfallsrichtung des wirksamen Strahlenkegels, 
wie sie z. B. dem Uebergang von der geraden zur schiefen Beleuchtung entsprechen, müssen dadurch herbeigeführt werden, dass man den Spiegel selbst seitlich gegen die Axe des Instruments bewegt und seine Fläche durch geeignete Drehung gegen die Lichtquelle wieder von Neuem leuchtend macht. Dabei ist uberdies eine Beleuchtung des Präparats durch a uffallende Strahlen, so wie überhaupt jede Beobachtung rein positiv ex Biller der mikroskopischen Objecte in dunklem Gesichtsfeld zunächst ganz ausgeschlossen und auch unter Mithilfe des Lieberkühn'schen Spiegels nur in schwerfälliger und höchst mangelhafter Form möglich. - Andrerseits bleiben für die Regulirung der Beleuchtung im Punkte der Quantität nur die beiden Hilfsmittel: die Annäherung und Entfernung des Spiegels, durch welche die lichtgebende Fläche für das Object grösseren oder geringeren angularen Durchmesser gewinnt; und sodann die Anwendung besonderer Diaphragmen unter dem Präparat, um durch diese den wirksamen 'Theil der Spiegelfläche beliebig zu beschräuken. Die erstere Methode, deren Anwendung an vielen Stativen darin vorgesehen ist, dass man den Spiegel an seinem Träger verschiebbar gemacht hat, gewährt nur einen geringen Spielraum; Diaphragmen aber lassen sich in derjenigen Form, die allein eine sichere Regulirung gestattet (nämlich als verschiebbare Cylinderblendungen), ohne grosse Unbequemlichkeit nur für rein centrale Beleuchtung verwenden.

Hier - und hier ganz allein - ist das Feld, auf welchem ein complicirter Beleuchtungsapparat der gewöhnlichen einfachen Vorrichtung möglicher Weise Concurrenz machen kann.

Es leuchtet nämlich sogleich ein, dass eine sehr viel einfachere und sicherere Regulirung und ein sehr viel grösserer Umfang in der möglichen Abstufung des Lichteinfalls zu erreichen wäre, wenn man am Orte des Objects eine Lichtstrahlung herstellen wollte, vermöge welcher dieses gleichzeitig a s allen Richtungen Licht empfängt, d. h. wenn man statt einer eng begrenzten lichtgebenden Fläche, wie sie der Spiegel gewährt, eine solche gewinnen könnte, welche das Object in sehr grossem Winkelraun von unten und der Beobachtung im auffallenden Lichte wegen - theilweise auch von oben umgiebt. Denn dann wären alle die verschieden gelegenen und verschieden grossen begrenzten Lichttlächen, welche für die einzelnen Beleuchtungsweisen $\mathrm{n}$ ach einander nöthig werden, $\mathrm{neben}$ einander gleichzeitig disponibel. Jede einzelne brauchte, wenn sie 
verlangt wird, nicht immer wieder neu hergestellt zu werden, sondern es würde statt dessen nur eine Abblendung der nicht in Anspruch zu nehmenden Theile auszuführen sein.

Das ist die Idee, auf deren Realisirung der hier zu beschreibende Illuminator ausgeht. Die Möglichkeit aber, das Geforderte in dem ganzen angegebenen Umfang mit relativ einfachen. Mitteln wirklich zu leisten, wird sogleich ersichtlich, wenn man die Lichtstrahlung betrachtet, welcher ein Object im Brennpunkt eines Linsensystems von grossem Oeffnungswinkel ausgesetzt ist. Wemn ein solches, sei es direct, sei es durch Vermittelung eines ebenen Spiegels, das Bild einer entfernten Lichtquelle, etwa einer Wolke, auf oder nahe dem Object entwirft, wird letzteres Punkt für Punkt so bestrahlt, als ob vor - d. h. bei der gewöhnlichen Anordnung am Mikroskop, unter - demselben eine gleichmässig leuchtende Fläche ausgebreitet wäre, deren Leuchtkraft, von den Lichtverlusten abgesehen, derjenigen der primären Lichtquelle gleich ist und deren scheinbare Grösse für das Object unmittelbar durch den Oeffnungswinkel des Linsensystems für den betreffenden Brennpunkt sich bestimmt. Man braucht diesen Oeffnungswinkel —- was keinerlei Hinlerniss findet - nur auf volle 180 Grad zu steigern, um eine Lichtquelle disponibel zu haben, die das Object von unten grade so wie das gleichförmig leuchtend gedachte Himmelsgewölbe einen im Freien stehenden Gegenstand von oben amspannt. Es ist klar, dass aus dieser die ganze Halbkugel bedeckenden Lichtfläche jeder beliebig begrenzte und beliebig gelegene Theil für sich in Wirkung gesetzt werden kann, wenn man den Eintritt der Lichtstrahlen in das Linsensystem durch ein Diaphragma mit entsprechendem Ausschnitt vor der dem Präparat gegenüber liegenden freien Oeffnung begrenzt; und dass sich durch blosse Veränderung der Grösse und der Lage jenes Ausschnittes, ohne alle sonstigen Eingriffe, alle möglichen Abstufungen zunächst der Beleuchtung mit durchfallendem Lichte herbeiführen lassen. Das Bild des Diaphragmas nämlich, welches die Linsen nach der Seite des Objects hin entwerfen, giebt die Begrenzung der wirksam werdenden Lichtfläche. Ein Diaphragma mit weitem Ausschnitt (relativ zum Durchmesser der ganzen freien Deffnung) liefert Strahlenkegel von grossem Dive1genzwinkel; ein enger Ausschnitt giebt beliebig verengte Strahlenbüschel. Steht das Diaphragma central, so giebt der durch seine Oeffnung wirksam werdende Theil der ganzen disponibeln Lichtfläche die 
gewöhnliche gernde Beleuchtung; wird dagegen die Oeffnung in irgend einem Azimuth mehr oder minder excentrisch gestellt, so erhält man alle Abstufungen dex schiefen Beleuchtung innerhalb dieses Azimuths. Damit ist aber der Umfang möglicher Beleuchtungseffecte noch keineswegs erschöpft. Denn wenn das Präparat im Focus des angenommenen Linsensystems nach dem Mikroskop hin durch eine durchsichtige Schicht mit syiegelnder oberer Grenzfläche bedeckt ist - wie das Deckglas immer ganz von selbst herbeiführt - so werden alle Strahlen, welche durch durchsichtige Theile des. Präparats hindurchgetreten sind, an dieser obern Grenzfläche partiell reffectirt, und fallen von o ben wieder auf das Object herab. Es ist leicht zu beweisen, dass der gesammte hieraus resultirende Beleuchtungseffect sich in allen Stücken genau so gestaltet, wie wenn ausser der vorher betrachteten lichtstrahlenden Halbkugel unter dem Präparat eine eben solche - nämlich ihr vom Deckglas nach oben entworfenes Spiegelbild - oberhalb des Präparats, jedoch mit sehr verminderter Leuchtkraft, wirksam wäre. - Soll die so erzengte diffuse Beleuchtung zur mikroskopischen Beobachtung des Objects in einem positiven Bille verwandt werden, so ist nichts weiter nöthig, als dass derjenige Theil der untern Lichttläche, welcher direct Lichtstrahlen in das Milkroskop senden wärde, unwirksam gemacht werde. Dies geschieht aher durch eine einfache Centralblende vor der freien Oeffnung der Beleuchtungslinsen, deren Durchmesser so gross genommen werden muss, dass der ganze dem Oeffnungswinkel des Mikroskops entsprechende centrale Strahlenkegel ausser Thätigkeit kommt, das freie Gesichtsfeld also vollkommen dunkel wird. Die zun Präparat gelangenden Strahlen sind dann ausschliesslich diejenigen von der äussersten peripherischen Zone der freien Oefhung des Beleuchtungssystems und der dieser entsprechenden strahlenden Halblkugel. $\mathrm{Da}$ es diejenigen sind, welche nach ihrem Durchtritt durch lichte Stellen des Präparats wegen ihres schiefen Einfalls am stärksten von der obern Deckglasfläche reflectirt werden, so tragen sie am meisten zur Beleuchtung des Objects von oben bei. - Aus dieser Beleuchlung resultirt eine mehr oder minder kräftige diffuse Lichtstrahlung, welche, so weit sie innerhalb des Oeffnungswinkels des Objentivs verläuft, ein positives Bild des Präparats auf dunklem Grunde erzengt. Selbstverständlich aber tragen zu diesem auch alle diejenigen Strahlen bei, welche durch Brechung oder durch Beugung im Präparat selbst 
nach der Axe des Mikroskops hin abgelenkt werden und dadurch direct in das Objectiv eintreten können.

Die hier gegebene Erklärung über die Strahlungsverhältnisse im Focus eines Linsensystems von grossem Oeffnungswinkel umfasst alle realen Wirkungen, welche ein sog. Condensor möglicher Weise gewähren kann und zugleich den Beleuchtungseffect durch das Wenham'sche Paraboloid. Was darüber hinausliegt - der ganze Begriff einer Concentration oder Condensation des Lichts und alle die souderbaren Wirkungen, welche sich daran knüpfen sollen - erweist sich einer strengeren theoretischen Kritik als willkürliche Erfindung; die vermeintlichen Eriolge aber, welche man mit Condensoren erzielt haben will, stellen sich, soweit sie nicht im Vorstehenden ihre Begründung finden, bei accuratem Experimentiren als blosse Fabeln heraus.

Dass die "Condensor" oder "Illuminator" benannten künstlichen Beleuchtungsapparate bisher fast nur illusorische Ziele verfolgt haben und die realisirbaren Vortheile solcher Combinationen bei ihnen desshalb entweder gar nicht oder doch nur sehr verkümmert zur Geltung kommen konnten, erklärt den wohlverdienten Misscredit, in welchem dieselben bei fast allen Beobachtern stehen, die mit dem Mikroskop ernstlich arbeiten wollen. Die Sache liegt aber anders, wenn eine solche Beleuchtungsvorrichtung ihrer ganzen Construction nach darauf angelegt ist; mit den einfachsten Mitteln und in der vortheilhaftesten Form diejenigen Leistungen zu erzielen, welche, dem Obigen zufolge, ihren alleinigen Wirkungskreis ausmachen: möglichst grossen Spielraum in der Abstufung der Beleuchtung nach Qualität und Quantität und möglichste Sicherheit und Leichtigkeit der Regulirung. Nach dieser Seite hin lassen sich, trotz der unvermeidlichen Einbusse in der specifischen Intensität der Wirkungen - die übrigens in den meisten Fällen nur sehr gering zu sein braucht - Vortheile erreichen, deren Realität nicht bestritten werden kaun, in Bezug auf welche die Frage vielmehr nur die sein wird: wie hoch oder wie gering sie der Einzelne für seine speciellen Bedürfnisse anschlagen mag.

Alles weitere ergiebt die folgende Beschreibung des nach meinen Anguben ausgeführten Apparates.

Zwei unachromatische Linsen, A nebenstehender Figur, bilden das zur Beleuchtung dienende System, also den Condensor - um die einmal eingebärgerte Benennung beizubehalten. Es hat die Form? 


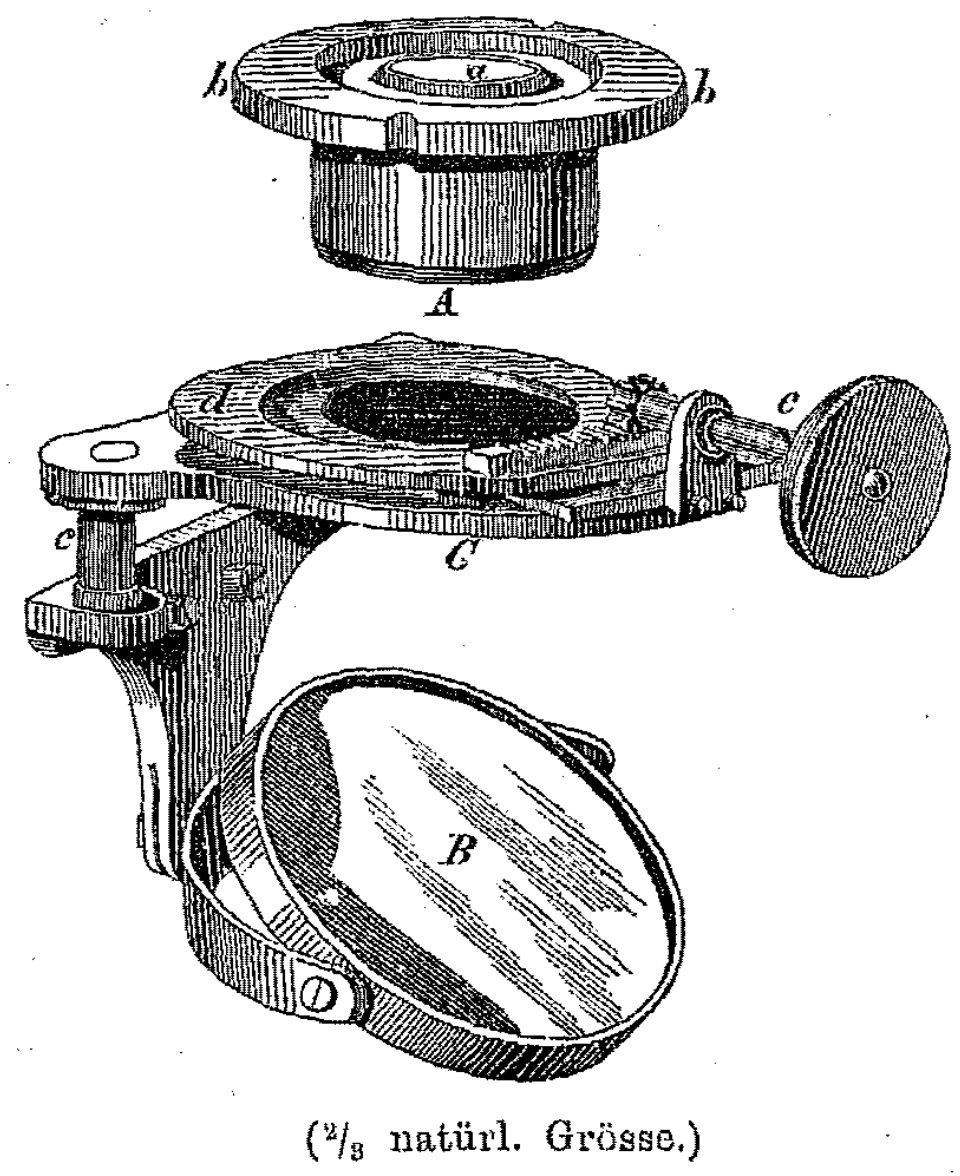

eines grossen Mikroskopobjectivs mit dicker, mehr als halbkugeliger, planconvexer Frontlinse, und ist in eine runde Messingscheibe bb gefasst, mit der es von oben in den Tisch des Miliroskops eingepasst wird. Es bleibt, weun einmal zum Gebrauch eingesetzt, ganz unbeweglich in der Axe des Instruments. Die nach oben gekehrte plane Fläche a der Frontlinse liegt dabei nur um eine Spur tiefer als die Ebene der Messingsscheibe bb, und da diese ihrer'seits genau in die Tischebene zu liegen kommt, so erleidet letztere keinerlei Unterbrechung, abgesehen von einer schmalen und flachen, die Linsenfläche ungebenden Rinne. Die Brennweite der ganzen Linsencombination beträgt ca. $15 \mathrm{Mm}$; erheblich grösser kann dieselbe - wiewohl solches an sich vortheilhaft wäre - nicht genommen werden, ohne auf unbequeme Dimensionen zu kommen. Der obere Brennpunkt aber liegt nur ein paar Millimeter über der planen Fläche der Frontlinse. Wenn daher ein Objectträger mit dem Präparat auf den Tisch des Mikroskops gebracht wird, so kommt das Präparat von selbst nahe in den Brennpunkt zu liegen, wobei die Substanz des Objectträgers, abgesehen von dem dünnen Zwischenraum unter ihm, die Fortsetzung der obein Condensorlinse bildet. - Wenn es in einzelnen Fälien clarauf ankommt, Lichtverluste möglichst zu vermeiden, kann man jenen Zwischenraum noch durch einen Tropfen Wasser ausfüllen.

Der Condensor ist anachromatisch, weil es für die beabsichtigte Wirkung absolut werthlos wäre, die als Lichtquelle dienende Wolke oder dergl. scharf abbilden zu wollen; wie es auch durchaus gleichgültig bleibt, ob das Bild genau im Nivenu des Präparats oder etwas darunter oder darüber entsteht. Dagegen ist darauf Bedacht ge- 
nommen, ihm für den obern Brennpunkt einen möglichst grossen Oeffuungswinkel zu geben. Er liefert - namentlich zum Zweck der Erzengung lichtstarker positiver Bilder - noch Strahlen, welche in einer W a sser schicht bis nahe $60^{\circ}$ gegen die Axe geneigt sind, welche also - da der Grenzwinkel der Totalreflexion für Wasser ca. $48^{\circ}$ ist - aus einem Luftraum dem Präparat niemals zugeführt werden könnten. Sollen aber Strahlen von solcher Schiefe für irgend einen Zweck in Wirlssamkeit treten, so darf natürlich das Präparat sich nicht in Luft befinden, wio auch der Zwischenraum unter dem Objectträger nicht durch Luft, sondern durch Wasser ausgefüllt sein muss.

Die Strahlen der primären Lichtquelle werden dem Condensor durch einen Planspiegel $B$, der nur um einen festen Punkt in der Axe des Instruments drehbar, nicht seitlich beweglich ist, zuggeführt. Die Regulirung der Beleuchtung aber vermittelt ein besonderer Diaphragmenträger $\mathrm{C}$ einige Centimenter unter dem Tisch des Mikroskops zwischen Planspiegel und Condensor, nahe dem untern Brempunkte des letztern. Die durch verschiedene Diaphragmen aus der gesammten disponibeln Lichtfläche ausgesonderten wirksamen Theile verhalten sich demnach, dem Object gegenüber, wie sehr entfernte aber entsprechend ausgedehnte leuchtende Flächen. Um die Blendungen schnell und sicher wechseln zu können, ist der ganze Diaphragmenträger um einen seitlich stehenden Zapfen $c$ drehbar, lässt sich durch diese Drehung unter dem Tisch des Mikroskops hervorbewegen, so dass er bequem zugänglich wird, und mit Einer Handbewegung wieder in die richtige centrale Stellung zurückschlagen. Zur Aufnahme der Blendungen dient nicht dieser Träger unmittelbar, sondern eine auf ihm drehbare und verschiebbare Scheibe $d$, deren Bewegung, nachdem das Ditphragma in eine Oeffnung in ihr eingelegt und der ganze Träger unter den Tisch zurückgeschlagen ist, alle Modificationen in der Richtung der einfallenden Lichtstrahlen ausführt. Drehung und Verschiebung werden gleichzeitig mittelst eines unter dem Tisch hervortretenden Griffes mit ränderirtem Knopf e bewirkt; in der Art, dass dieser Griff, zwischen den Fingern um seine eigene Axe gedreht, durch /ahn und Trieb die Scheibe mit sammt der Blendung in radialer Richtung verschiebt, während die auf diese Weise excentrisch gestellte Diaphragmenöffnung im Umfang von ca. $120^{\circ}$ um die Axe des Mikroskops herumgeführt werden kann, wenn der Griff als Hebel einer 
horizontalen Drehung benutzt wird. - Die Mitte im Spielraum der radialen Verschiebung, die der Trieb gewährt, $d$. $h$. die centrale Stellung des einliegenden Diaphragmas, deutet sich dem Finger durch das Einspringen eines federnden Zahnes sicher an. - Der Knopf des Triebes dient zugleich als Handhabe für das Vor- und Zurückschlagen des ganzen Trägers.

Was zunächst die Benutzung des Apparates zur gewöhnlichen Beobachtung mit durchfallendem Licht anlangt, so dient ihr eine Anzahl kreisförmiger Scheiben von ca. $30 \mathrm{Mm}$. Durchmesser mit concentrischen Oeffnungen von $1-7 \mathrm{Mm}$. Weite. Bei centraler Stellung, des Triebes gewähren sie, nach einander eingelegt, alle wünschenswerthen Abstufungen der gewöhnlichen geraden Beleuchtung. Zwar wird dabei keine stetige Veränderung in der Oeffnung des einfallenden Lichtkegels, wie bei einer zurückziehbaren Cylinderblendung, herbeigeführt; da indess ihre lichten Durchmesser einander beliebig nahe liegen können, und wern man sich an die paar Handgriffe gewöhnt hat, das Wechseln dieser Diaphragmen ausserordentlich schnell geschieht, so erscheint für den gewöhnlichen normalen Gebrauch des Mikroskops diese Einrichtung, verglichen mit dem Oberhüuser'schen Blendungsapparat, keineswegs im Nachtheil; zumal grade für die centrale Beleuchtung der Lichtverlust im Condensor unwahrnehmbar klein bleibt. - Der Uebergang vom geraden zu beliebig suhiefem Licht erfolgt aber mit jeder Grösse des wirksamen Strahlenkegels bloss durch Handhabung des erwähnten Triebgriffes, ohne alle Nachhilfe am Spiegel; und zwar hat man es in der Gewalt, auf die angegebene Weise nicht nur die strahlende Fläche bis an die Grenze der freien Oeffnung der starken Objective aus der Axe zu entfernen, sondern zugleich auch - durch Drehung des excentrischen Diaphragmas im Azimuth - das schiefe Licht von jeder Seite her gegen das Object einfallen zu lassen; daher denn bei dem beschriebenen Illuminator die Rotation des Mikroskops um seine Verticalaxe für diesen Zweck entbehrlich wird. - Die Regulirung der schiefen Beleuchtung wird auf dicse Weise ausserordentlich leicht und sicher ausführbar; besonders hat man den Vortheil, von jeder Modification zu jeder andern nach Belieben übergehen zu können, ohne immer von Neuem nach dem richtigen Licht suchen zu müssen. Wenn der Spiegel ein mal so eingestellt ist, dass der Condensor die volle Beleuchtung gewährt, so bleibt diese so lange bestehen, als die Lichtquelle sich nicht ändert. - 
Dagegen muss aber ausdrücklich hervorgehoben werden, dass für sehr schiefes Licht - wenn es sich etwa darum handelt, an Testobjecten die äusserste Grenze des Auflösungsvermögens der Objective in Anspruch zu nehmen, - der Apparat nicht ganz das erreicht, was der einfache Hohlspiegel leisten kann. Der. Grund liegt theils darin, dass die stark geneigten Strahlen eine merkbare Verminderung ihrer Intensität erleiden; theils ist er in dem Umstande zu suchen, dass die unvermeidlichen Reflexe an den Flächen des Condensors bei excentrischer Lage der Diaphragmenöffuung Nebenbilder der Lichtfläche unter dem Präparat erzeugen, deren Beitrag zum mikroskopischen Bilde die Wirkung beeinträchtigen muss. Es dürfte - ausser durch ganz unpraktische Complicationen - nicht möglich sein, diesen Nachtheil zu beseitigen. Da er aber bei centraler Beleuchtung vollkommen hinwegfällt, weil alsdann alle Reflexbilder in der. Axe liegen, bei mässig schiefem Lichteinfall er aber auch unmerklich bleibt, der geringen Intensität der Reflexe wegen, so leidet darunter der gewöhnliche Gebrauch des Mikroskops nicht im geringsten. Nur scheint es, im Hinblick auf den namhaft gemachten ungewöhnlichen Fall, angemessen, die Einrichtung so zu treffen, dass der Illuminator ohne Umstände mit dem einfachen Hohlspiegel vertauscht werden kann. Es wird desshalb der ganze Diaphragmenapparat mit dem zugehörigen Planspiegel zu einem Stück verbunden, welches (mittelst einer Coulisse) ebenso schnell und sicher unter dem 'Tisch des Mikroskops angebracht und wieder entfernt, wie der Condensor in die Tischplatte eingefügt und wieder ausgehoben werden kaun.

Soll mit polarisirtem Licht beobachtet werden, so braucht nur der polarisirende Nicol, in eine passende Metallscheibe gefasst, an Stelle einer Blendung in den Diaphragmenträger eingesetzt zu werden. Die Wirkung ist in allen Stücken die nämliche wie bei der gewohnten Einrichtung.

Was endlich die Beobachtung der Objecte durch positive Bilder in dunklem Felde anlangt, so tritt der betreffende Beleuchtungseffect in der oben erläuterten Form ohne alles weitere Zuthun in Thätigkeit, sobald in den Diaphragmenträger bei centrischer Stellung desselben statt der früheren Lochblendungen ein schmaler Ring eingelegt wird, der mittelst dünner Speichen eine centrale Scheibe von ca. $12 \mathrm{Mm}$. Durchmesser trägt. Ist der Oeffnungswinkel des angewandten Objectivs kleiner als der angulare Durchmesser des 
auf diese Weise unwirlisam gemachten mittleren Theils der disponibeln Lichtfläche unter dem Präparat, so gelangt kein geradlinig durch letzteres hindurchtretender Strabl in das Mikroskop und das freie Sehfeld wird vollkommen dunkel. Das Bild wird ausschliesslich durch solche Strahlen erzeugt, die nach dem Durchtritt durch das Präparat an der Grenze zwischen Deckglas und Luft durch Spiegelung auf jenes herabgeworfen und an seiner Oberfläche wieder diffus reflectirt sind und durch solche, welche auf ihrem Wege durch das Priparat, sei es durch Brechung, sei es durch Beugung, nach der Axe hin abgelenkt worden sind. Je uach der Structur der Objecte ist bald der eine, bald der andere Theil der überwiegende; bei Verwendung einer Immersionslinse bleibt natürlich nur das im Object abgelenkte Licht wirksam, da die Reflexion an der obern Deckglasfläche fast ganz fortfüllt. Ist das Präparat weder allzu durchsichtig noch allzu undurchsichtig und sind sonst die Umstände fiir die fragliche Wirkung günstig, so können die so erhaltenen rein positiven Bilder eine beträchtliche Lichtstärlke gewinnen. In vielen Fällen - z. B. bei trocken liegenden Diatomeen - ist die Helligkeit gross genug, um bei gewöhnlichem guten Tageslicht eine 5-600fache-Vergrösserung recht wohl zu gestatten, namentlich dann, wenn der Zwischenraum zwischen der obern Condensorlinse und dem Objectträger durch einen Tropfen Wasser ausgefüllt wird.

Sollen mit dieser Beleuchtungsart Objective verwandt werden, deren Oeffnungswinkel merklich aber $40^{\circ}$ hinausgeht, so muss derselbe durch Abblendung der peripherischen Zone entsprechend reducirt werden; einestheils, weil sonst das Gesichtsfeld nicht dunkel erhalten werden kann, ohne die wirksame Lichtfiäche im Condensor allzu sehr zu schmälern; anderntheils, auch desshalb, weil ein zu grosser Divergenzwinkel der abbildenden Strahlenkegel fast bei allen Objecten die Vollkommenheit des Bildes sehr beeinträchtigt. - Diese bei allen stärkeren Objectiven unentbehrliche Abblendung wird durch Diaphragmen mit passender Oeffnung, die man über die oberste Linse aufschraubt, bewirkt. Das Auflösungsvermögen des Objectivs wird dadurch natürlich auf dasjenige Maass vermindert, welches der übrig bleibenden freien Oeffnung entspricht.

Ich rnus es dahin gestellt sein lassen, ob und in wie weit diese Beleuchtungsart - welche im. Wesentlichen die des Wenham'schen Paraboloids' ist - für wissenschaftliche Untersuchungen irgend eine Bedeutung gewinnen kann. Jedenfalls aber unterliogt sie bei der hier in 
480 Dr. E. A b b e: Ueber einen nenen Beleúchtungsapparat am Mikroskop.

Rede stehenden Einrichtung nicht im geringsten den Ausstellungen, die man in Bezug auf die Vollkommenheit der Bilder bei ihr erhoben hat und die Harting als dem Princip dieser Wirkung anhaftend hinstellt. Im Gegentheil erhält man mit guten Objectiven auch bei starker Vergrösserung - wenn sonst die Objecte darnach sind - nicht nur sehr nette und deutliche, sondern häufig auch sehr charakteristische Bilder, die wegen ihres plastischen Hervortretens auf dem dunklen Grunde mindestens für Demonstrationen oft entschiedene Vortheile bieten dürten.

In Bezug auf den Gebrauch des Apparates mit durchfallendem Licht mag noch bemerkt sein, dass bei Anwendung sehr schwacher Vergrösserungen, wenn die disponible Lichtquelle nur wenig ausgedehnt ist, häufig keine gleichförmige Beleuchtung des Objects erhalten wird, weil das Bild der Lichtquelle im Focus des Condensors nicht gross genug ist, um den ganzen sichtbaren Umfang des Präparats zu bedecken. Man gewinnt in diesem Falle ein gleichmässig und hinreichend stark erhelltes Sehfeld, wenn man den Planspiegel durch ein Stück weisses Papier überdeckt. - Soll mit Lampenlicht beobachtet werden, so ist es das Zweckmässigste, in gerader Linie zwischen Flamme und Spiegel eine möglichst grosse Sammellinse am einfachsten eine mit mässig blau gefärbtem Wasser gefüllte grosse Glaskugel - einzuschalten, um die Leuchtlkraft der kleinen Flamme auf deren grössere Oberfläche zu übertragen. Dieses ist erreicht, wenn die Spitze des von der Linse oder der Kugel ausgehenden Lichtkegels den Spiegel trifft und dessen Fläche vollständig erhelit.

Schliesslich sei noch darauf hingewiesen, dass die Beobachtung des im vorangehenden Aufsatze mehrfach erwähnten Oeffnungsbildes in der obern Focalebene des Objectivs (durch Herabsehen in den offenen Tubus) das einfachste Mittel darbietet, alle hier discutirten Wirkungen direct zu controliren und dass eine solche Controle eben so sehr für die praktische Handhabung des in Rede stehenden Apparates wie für die richtige Beurtheilung der verschiedenen Beleuchtungseffecte überhaupt von Vortheil sein wird. 